\title{
Design and Implementation on Concert Live System for iOS Platform
}

\author{
Luo Luo \\ Department of Music \\ Xi'an Shiyou University \\ Xian, P.R. China \\ art_renjia@163.com
}

\begin{abstract}
Music is the key to open the door of the soul with infinite charm, and it is an important form of meeting people's spiritual and cultural needs. For people watching a concert by time and place restrictions and other issues, this paper based on iOS platform design concert live system. Firstly, research the iOS platform architecture, it is composed by cocoa touch layer, media application layer, core service layer, sore OS layer and other layers; then, studies streaming media encoding technology, audio coding using AAC technology, video encoded using H.264 technology; finally, construct the software development platform, designed HLS logical flow and to study the technical key points. The design scheme of this paper is based on the data real time transfer mechanism, has strong network adaptability, and can lighten the workload of server and client.
\end{abstract}

Keywords- iOS platform; concert; live system; design and implementation

\section{INTRODUCTION}

Music is one of the most ancient and most universal and infectious art forms of human, is an important means that the creator through the specific sound structure to achieve thought and emotional expression and communication, is an organic part of human spiritual life, as an important form and carrier of human culture, contains a wealth of cultural and historical connotations, with its unique way to satisfy the spiritual and cultural needs of the people, to music perception is a kind of response of human basic qualities [1]. With the economic and social development, the quality of people's material life is continuously improving, while, on the spiritual and cultural life also put forward higher requirements. The concert is one of the ways to meet people's spiritual life [2]. Concert, also known as recitals, refers to the live performance in front of the audience, is usually musical performance, can be a performed by the individual musicians, and can also be collective performances of music groups. The venue of the concert included the public performing arts hall, the nightclub, the music house, the gymnasium, the barn, the concert hall and the multifunctional performance place and so on. Limited by the venue space and venue, to watch the concert live just a few people, cannot meet the music needs of most people.

If the concert live mobile live, people through mobile communications equipment to listen or watch, solved the real problem of the traditional concert only a few people enjoy. iOS (i Operating System) was mobile operating system developed by Apple Corp, it belongs to the commercial operating system of Unix class, originally designed and developed for the iPhone, then gradually applied to Apple's iPod touch, iPad and Apple TV and other products. iOS programming interfaces, tools, and resources are all included in the iOS SDK, developers with an integrated development environment complete the application development [3-4]. Apple's iPad, iPhone and other mobile products with high market share, the paper based on iOS platform designed concert live system, for software development to provide solutions and technical support, for the majority of Apple mobile device users to provide the most advanced music information services.

\section{IOS PLATFORM HIERARCHY}

The iOS platform is composed of 4 layers, each layer containing several components, and the corresponding function is completed by the component. The platform hierarchy is shown in Figure 1.

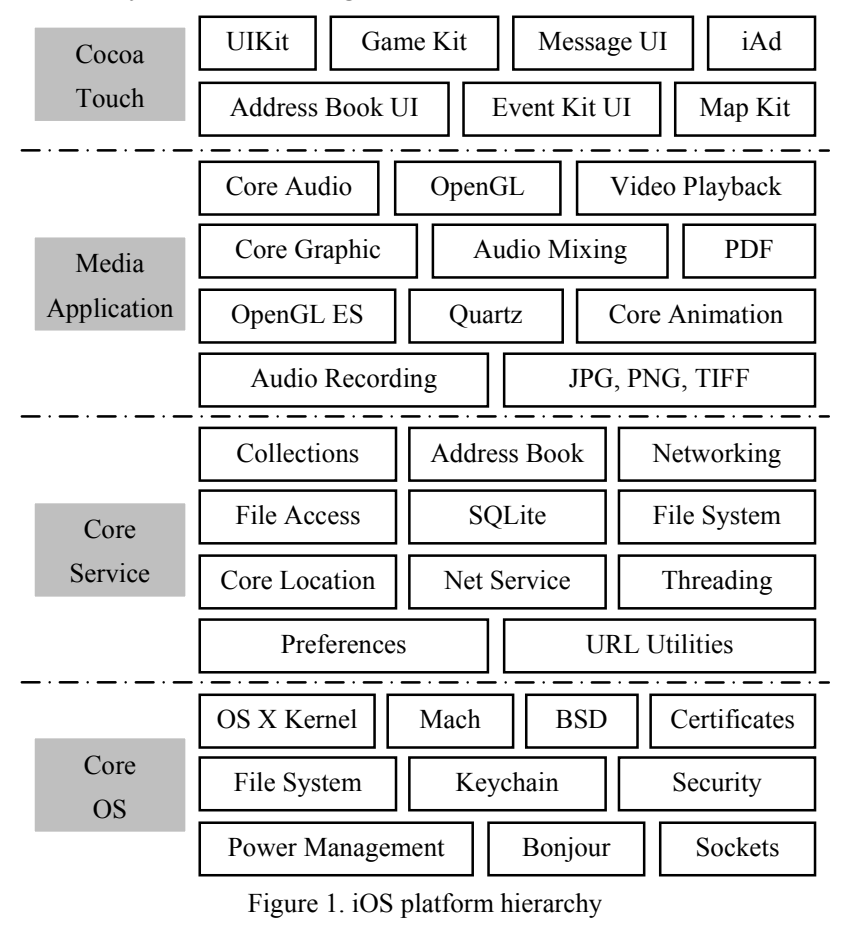
7]: 
- Cocoa Touch layer. Through the interface control, multi task and multi point touch and other key technologies, providing realize human-machine interaction, touch operation application, provides a convenient method and technical support for the development of all types of intelligent terminal software. The UIKit framework is the core part that contains the Objective- $\mathrm{C}$ program interface, to provide the implementation of various components of the program interface, the key architecture for event driven iPhone applications.

- Media Application layer. Provide a combination of each other, can complete various images, audio and video and other multimedia services application and related development technology, for mobile devices to bring the perfect multimedia experience. Application program using various media files, for audio and video recording, graphics rendering, as well as the production of simple animation.

- Core Services layer. Located on above the Core OS layer, provides basic system services for all applications program. The main framework include: CoreFoundation framework for iOS application develop basic data management and service functions; CFNetwork framework provides object-oriented abstraction of network protocol; CoreLocation framework leverages nearby GPS, cellular base stations or Wi-Fi signal information measuring user's current location.

- Core OS layer. Located at the bottom of the iOS hierarchy, including the operating system kernel environment, drivers and basic interface, API functions provided by the $\mathrm{C}$ language completed the interaction with hardware and some other operational tasks, including Bonjour and DNS services, hardware drivers, the regional language information, memory allocation, program management, thread management, mathematics computing, file access, network management, standard input and output.

\section{Audio CODING TECHNOLOGY}

Audio coding technology is the use of AAC (Advanced Audio Coding), which is a file compression format designed specifically for sound data, unlike MP3, using the new algorithm for encoding, more efficient, with more high "cost-effective". The use of AAC format, make people feel the sound quality is not significantly reduced under the premise, more compact.

AAC appeared in 1997, is based on MPEG-2 audio coding technology is. Co-developed by Fraunhofer IIS, Dolby Laboratories, AT\&T, Sony and other companies, intended to replace the MP3 format. In 2000, after the emergence of MPEG-4 standard, AAC reintegrated its characteristics, joined the SBR technology and PS techniques, in order to distinguish it from the traditional MPEG-2 AAC, also known as MPEG-4 AAC. 2003 HE-
AAC V2 officially became a part of the MPEG-4 audio standard, is currently the most effective audio coding techniques. AAC technology development process is shown in Figure 2 [8].

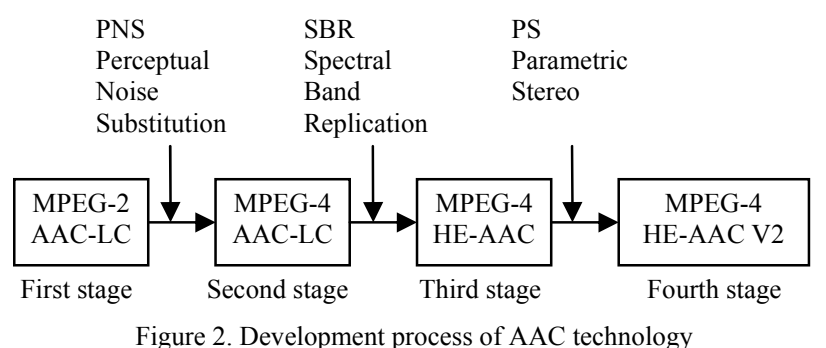

HE-AAC V2 is by the three kinds of technology of MPEG-4 audio standard technology, AAC (Advanced Audio Coding) and SBR (Spectral Band Replication) and PS (Parametric Stereo), with the formation of a new audio coding method.MPEG-4 AAC is provided an equally good method of encoding and decoding for all audio material, like other MPEG audio coding method, AAC also on the energy component of the audio signal analysis and the use of the human ear "masking effect", in order from the audio signal find hearing can be other sound mask component. MPEG-4 SBR is a band-spreading tool, joint work with the original audio encoder for broadening the audio bandwidth, so the audio encoder at half the bit rate transmission with the same quality of the audio signal. MPEG-4 PS is a compression coding method for stereo audio signal, in order to further improve the efficiency of the low-rate stereo coding provides a new approach. AAC coding principles and characteristics are described below:

- AAC is a high compression ratio of audio compression algorithm, but its compression ratio to be far more than the older audio compression algorithms, such as AC-3, MP3 and so on. And its quality can be comparable with the non compressed CD sound quality.

- Like other similar audio coding algorithms, the AAC uses a transform coding algorithm, but AAC uses a higher resolution filter bank, so it can achieve higher compression ratio.

- AAC uses a temporary noise restructuring, backward adaptive linear prediction, joint stereo technique and quantization Huffman coding, and other latest technology, the use of these new technologies make compression ratio can be improved further.

- $\quad$ AAC support more kinds of sampling rate and bit rate, support 1 to 48 tracks, support up to 15 low frequency tracks, with a variety of language compatible ability, as well as many as 15 embedded data flow.

- AAC supports a wider range of sound frequencies, the highest of which can reach $96 \mathrm{kHz}$, the lowest up to $8 \mathrm{~Hz}$, and far wider than the range of MP3 $20-44 \mathrm{kHz}$. 
- Unlike the MP3 and WMA, the AAC has no loss of the very high and low frequency components of the audio frequency, and is more close to the original audio in the spectrum structure than the WMA, and thus the fidelity of sound is better. Professional evaluation indicated, AAC sounds clearer than WMA, and more close to the original sound.

- AAC uses optimized algorithms to achieve a higher decoding efficiency, requiring less processing power when decoding.

\section{Video Coding TeChNOLOGY}

H.264 is a highly compressed digital video codec standard proposed by the JVT (Joint Video Team), it is a new generation of digital video compression format. Encoding and decoding process includes five parts: intra frame and intra prediction, transform and inverse transform, quantization and inverse quantization, loop filter, entropy coding. The encoder is to achieve encoding device, the signal or data compiled, converted to a form of signal which is used to communications, transport and storage. The main features of the H.264 standard are described as follows:

- Higher coding efficiency. Compared with the bit rate efficiency of $\mathrm{H} .263$ standards, the code rate can save an average of more than $50 \%$.

- High quality video images. H.264 provides highquality video images at low code rates, and provides high quality image transmission is the highlight of H.264 application in the lower bandwidth.

- Improve network adaptability. H.264 can work in the low delay mode of real-time communication applications (such as video conferencing), it can also work without delayed video storage or video streaming server.

- Use hybrid coding structure. Is the same as the H.263, H.264 also use the DCT transform coding and DPCM differential encoding hybrid coding structure, but also adds multi-mode motion estimation, intra prediction, multi-frame prediction, variable length coding based on contents, $4 \times 4$ Two-dimensional integer transform and other new coding methods to improve the coding efficiency.

- Coding options of H.264 is fewer. When H.263 encoding often need to set up quite a few options, increasing the difficulty of coding, while H.264 achieved simple "back to basics", reducing the complexity of encoding.

- H.264 can be applied in different situations. H.264 can use different transmission and playback rate according to different environment, and provides a wealth of error handling tools, can well control or eliminate packet loss and error code.

- Error recovery function. H.264 provides a tool for solving the network transmission packet loss, and is suitable for transmitting video data in wireless network with high error code rate transmission.

- Higher complexity. H.264 performance improvement is obtained at the expense of increasing complexity. It is estimated that the computational complexity of H.264 coding is about 3 times that of H.263, and the decoding complexity is equivalent to approximately 2 times of H.263.

H.264 encoder principle is shown in Figure 3.

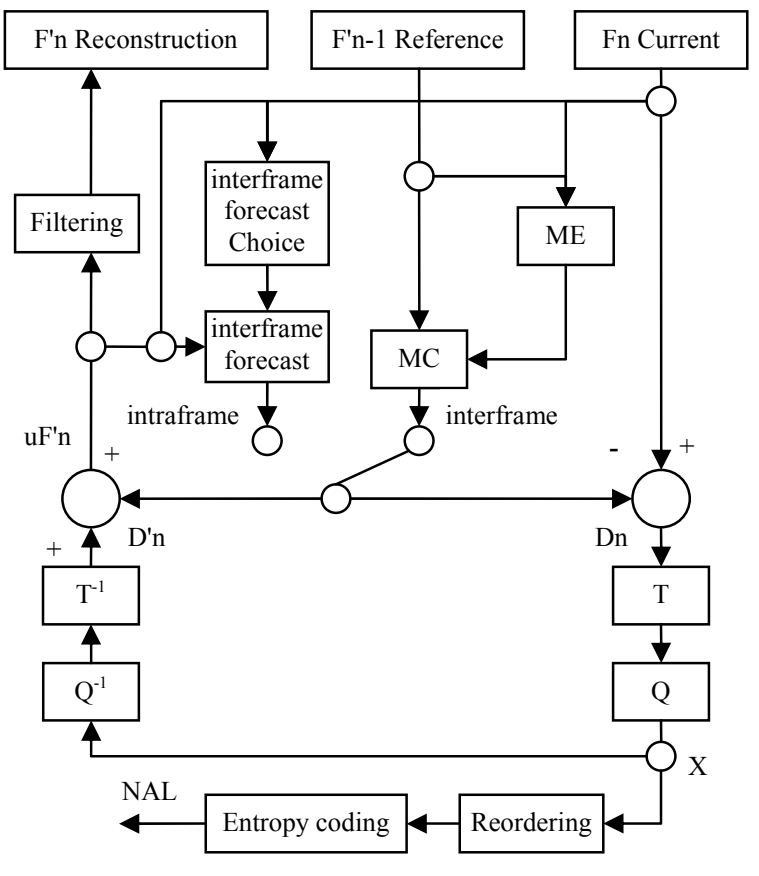

Figure 3. Encoder principle on H.264

In Figure 3, the encoder has two major data flow paths, "up $\rightarrow$ down" coding and "down $\rightarrow$ up" reconstruction. Principles is described as follow [9-11]: Take the macro block as the unit, the n-th frame Fn of the input video encoded; motion estimation function to find the current macro block matching area from the reference frame, the region and the current macro block position shift is the motion vector MV; according to MV generate motion compensated prediction $\mathrm{P}$; with the current macro block minus $\mathrm{P}$ is generated on the current frame residual macro block Dn; Dn is divided into $8 \times 8$ or $4 \times 4$ sub-block, respectively DCT transformation; sub-block quantization (X); DCT coefficient of sub-block reordering, then the run-level run-length encoding; coefficient coded, motion vectors and the macro block header information is generated after the entropy coding compression bit rate.

\section{CONSTRUCTION ON SOFTWARE DEVELOPMENT PLATFORM}

HLS (HTTP Live Streaming) is based on the HTTP protocol implementation proposed by Apple Inc, which is part of QuickTime X and iPhone software systems. 
Through HTTP protocol, the video and audio sent from the server to the iOS device, with the application of in-depth, but also extended to the Android system [12]. Working principle is to divide the whole stream into a small HTTPbased file download, is an asymptotic method of download.

Achieve HLS LiveEncoder encoder, logic flow is respectively open the audio and video coding thread through DirectShow technology to achieve audio and video capture, and then respectively call libx264 and libfaac to perform video and audio encoding. After two code threads coded audio and video data in real time, according to the custom of partitioning strategy, stored in a segmented file of MPEG-TS format, after the completion of segmented file storage, updating $\mathrm{m} 3 \mathrm{u} 8$ index file. The logic process is shown in Figure 4.

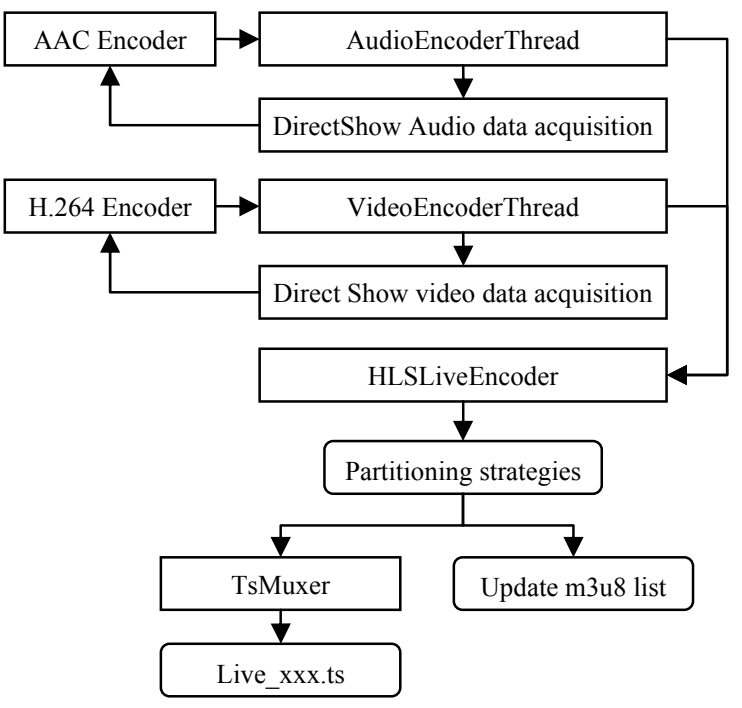

Figure 4. Logic process of HLS

Implement HTTP Live Streaming live, need to study and implement the key points of the following technologies:

- Capture video and audio source data. Use DirectShow for video and audio data real-time acquisition, DirectShow is Microsoft Corp based on ActiveMovie and video for Windows launched, a new generation of COM (Component Object Model) development package is part of DirectX, the use of multimedia streaming processing, support multiple file formats and compression format.

- Perform H.264 encoding for the original data. H.264 is a highly compressed digital video codec standard proposed by JVT (Joint Video Team). Encoding and decoding process mainly including five parts: inter frame and intra frame estimation, transform and inverse transform, quantization and inverse quantization, loop filter, entropy coding.

- Video and audio data is encapsulated for MPEGTS packages. MPEG2 defines the TransportStream (TS) and ProgramStream (PS).
MPEG2-TS are used for audio, video and data communications protocol, and are mainly used for real-time transmission of programs. The TS code has the strong ability of resisting the transmission error code, and the current MPEG-2 code stream in the transmission media basically adopts the packet format of TS code stream.

- HLS partitioning strategy and m3u8 index file. Due to caching and other problem, the index file will retain the last three slices address, in the form of a "sliding window", to be updated. m3u8 is live index file of HTTP Live Streaming, can be considered .m $3 \mathrm{u}$ format file, except that the $\mathrm{m} 3 \mathrm{u} 8$ file using UTF-8 character encoding.

\section{CONCLUSION}

Use the concert live system that constructed by this article, to watch the concert without restrictions of time and place, not only to bring the enjoyment of beauty, but also expand the visibility and social impact of the concert, with a broad market space for development. The design of this article is based on HTTP streaming transfer protocol, using real-time transfer mechanism of media data, reducing the workload of the server and the client. At the same time, most firewalls allow HTTP protocol to penetrate, with strong network adaptability [14]. However, the development should pay attention to the factors such as slice interval, video code rate, resolution ratio, network environment, and others. In order to avoid the defects of HLS in time delay, improve the live effect.

\section{REFERENCES}

[1] Xinxin Lv, " Development Course and Construction Ideas on College Music Appreciation Teaching Material," Science technology \& publishing, vol. 32, pp. 116-120, Nov 2013.

[2] Y. L Wei, "Design and implementation of technology programs live symphony concert," Audio \& Video Production, vol. 41, pp. 57-61, Apr 2014.

[3] Charanjeet Singh, A.J. Roy, Sinchita Roy-Chowdhuri, "Imagebased Cytopathology Reference App on iOS Plat-form," Journal of the American Society of Cytopathology, vol. 38, pp. 11-12, Jul 2014.

[4] LI Lei, LI Xiaoli, "Research on mobile learning resource design based on iOS handheld terminal," Chinese audio-visual education, vol. 35, pp. 93-97, Dec 2014.

[5] C. Liu, Q. Zhu, Kenneth A. Holroyd, Elizabeth K. Seng, "Status and trends of mobile-health applications for iOS devices: A developer's perspective," Journal of Systems and Software, vol. 84, pp. 2022-2033, Nov 2011.

[6] Jonathan Zdziarski, "Identifying back doors, attack points, and surveillance mechanisms in iOS devices," Digital Investigation, vol. 11, pp. 3-19, Apr 2014.

[7] P. Lou, X. P. Pang, S. T. Ai, "Development of Polar Mobile Information Platform Basec on Android and iOS," Chinese Journal of Polar Research, vol. 27, pp. 98 - 103, Jan 2015.

[8] Y. C. Zhang, G. R. Hu, "Coding theory and its application of HEAAC V2," Chinese cable TV, vol. 14, pp. 293 - 296, Mar 2006.

[9] Mithilesh Kumar Jha, Sumantra Dutta Roy, Brejesh Lall, "DEMDbased video coding for textured videos in an H.264/MPEG framework," Pattern Recognition Letters, vol. 51, pp. 30 - 36, Jan 2015 . 
[10] Y. D. Wu, Y. Sun, Z. D. Feng, et al., "A novel total variation based frame layer rate control algorithm for H.264/AVC," Journal of Visual Communication and Image Representation, vol. 25, pp. 879-890, May 2014.

[11] D. W. Xu, R. D. Wang, Y. Q. Shi, "An improved reversible data hiding-based approach for intra-frame error concealment in H.264/AVC," Journal of Visual Communication and Image Representation, vol. 25, pp. 410 - 422, Feb 2014.

[12] B. W. Zheng, X. X. Quan, L. Liu, Y. Sun, "HLS - Share: A Player Middleware Timely Sharing HTTP Live Streaming," Computer Applications and Software, vol. 31, pp. 1 - 4, Jan 2014. 